\title{
Melatonin Promotes Apoptosis of Oxaliplatin-resistant Colorectal Cancer Cells Through Inhibition of Cellular Prion Protein
}

\author{
JUN HEE LEE ${ }^{1}$, YEO MIN YOON ${ }^{2}$, YONG-SEOK HAN ${ }^{2}$, CHUL WON YUN ${ }^{2}$ and SANG HUN LEE ${ }^{2,3}$ \\ ${ }^{1}$ Department of Pharmacology and Toxicology, \\ University of Alabama at Birmingham School of Medicine, Birmingham, AL, U.S.A.; \\ ${ }^{2}$ Medical Science Research Institute, Soonchunhyang University Seoul Hospital, Seoul, Republic of Korea; \\ ${ }^{3}$ Department of Medical Bioscience, Soonchunhyang University, Asan, Republic of Korea
}

\begin{abstract}
Background/Aim: Drug resistance restricts the efficacy of chemotherapy in colorectal cancer. However, the detailed molecular mechanism of drug resistance in colorectal cancer cells remains unclear. Materials and Methods: The level of cellular prion protein $\left(\operatorname{PrP}^{C}\right)$ in oxaliplatin-resistant colorectal cancer (SNU-C5/Oxal-R) cells was assessed. Results: $\operatorname{PrP}^{C}$ level in SNU-C5/Oxal-R cells was significantly increased compared to that in wildtype (SNU-C5) cells. Superoxide dismutase and catalase activities were higher in SNU-C5/Oxal-R cells than in SNU-C5 cells. Treatment of SNU-C5/Oxal-R cells with oxaliplatin and melatonin reduced $\operatorname{PrP}^{C}$ expression, while suppressing antioxidant enzyme activity and increasing superoxide anion generation. In SNU-C5/Oxal-R cells, endoplasmic reticulum stress and apoptosis were significantly increased following co-treatment with oxaliplatin and melatonin compared to treatment with oxaliplatin alone. Conclusion: Co-treatment with oxaliplatin and melatonin increased endoplasmic reticulum stress in and apoptosis of SNU-C5/Oxal-R cells through inhibition of $\operatorname{PrP} P^{C}$, suggesting that $\operatorname{PrP}^{C}$ could be a key molecule in oxaliplatin resistance of colorectal cancer cells.
\end{abstract}

Colorectal cancer is one of the leading causes of cancerrelated deaths (1). Although improvements in surgical techniques are effective for patients with non-metastatic

Correspondence to: Sang Hun Lee, Ph.D., Soonchunhyang Medical Science Research Institute, Soonchunhyang University, Soonchunhyang University Seoul Hospital, 59, Daesagwan-ro (657 Hannam-dong), Yongsan-gu, Seoul 140-887, Republic of Korea. Tel: +82 027099029, e-mail: jhlee0407@sch.ac.kr

Key Words: Colorectal cancer cells, cellular prion protein, melatonin, oxaliplatin, drug resistance. colorectal cancer, half of all patients with colorectal cancer experience metastasis and recurrence. Therefore, chemotherapy is one of the choices for colorectal cancer therapy. Oxaliplatin, a bifunctional alkylating agent, can inhibit DNA replication and transcription by covalently binding to DNA, resulting in induction of apoptosis (2). However, drug resistance of cancer cells is a major limitation in chemotherapy (3). Although the mutation of anticancer drug target proteins, defective DNA damage repair, improved anticancer drug efflux, and alternative compensating signalling pathways are potential mechanisms of drug resistance, their effects in oxaliplatin-resistant cells remain unclear. Therefore, the identification of signalling pathways and key molecules associated with oxaliplatin resistance in colorectal cancer cells is of great importance for effective colorectal cancer chemotherapy.

Cellular prion protein $\left(\mathrm{PrP}^{\mathrm{C}}\right)$ is highly expressed and tethered to the cell surface by the glycosylphosphatidylinositol anchor in various cell types, including nerve cells (4). Although several studies have shown that $\operatorname{PrP}^{\mathrm{C}}$ regulates selfrenewal, differentiation, and functional enhancement in stem/progenitor cells (5-7), recent findings indicate that it is involved in cancer cell proliferation, cell survival, resistance to apoptosis, and metastasis (8-10). In addition, $\operatorname{PrP}^{\mathrm{C}}$ plays a critical role in the invasive capacity and the acquisition of multidrug resistance in cancer cells (11). Our previous study showed that $\operatorname{PrP}^{\mathrm{C}}$ is involved in 5-fluorouracil resistance in colorectal cancer cells by enhancing survival and proliferation through the phosphoinositide-3-kinase (PI3K) - RAC-alpha serine/threonine-protein kinase (AKT) signalling pathway (12). However, there is little evidence regarding any relationship between $\operatorname{PrP}^{\mathrm{C}}$ and oxaliplatin resistance in colorectal cancer cells.

Melatonin ( $N$-acetyl-5-methoxytryptamine $)$ is an endogenously secreted hormone produced by the pineal gland as well as several tissues including the bone marrow, 
gut, liver, ovaries, and testes (13). Generally, melatonin affects sleep, circadian rhythms, neuroendocrine actions, and immune reactions $(14,15)$. In addition, melatonin has antioxidant, anti-inflammatory, and apoptotic-regulatory effects $(16,17)$. Furthermore, increasing evidence has demonstrated the anticancer effect of melatonin (18). Melatonin has been reported to increase the level of reactive oxygen species (ROS), resulting in the decreased survival of HCT166 human colorectal carcinoma cells (19). Melatonin was also found to induce apoptosis of LoVo colorectal cancer cells in a dose-dependent manner (20). Furthermore, melatonin can induce cellular senescence and cell death in colorectal cancer cells by regulating apoptosis (21). These findings suggest that melatonin could be a potential therapeutic agent for colorectal cancer therapy.

In this study, we investigated the role of $\mathrm{PrP}^{\mathrm{C}}$ in oxaliplatin resistance of colorectal cancer cells by measuring the activity of antioxidant enzymes. Moreover, we also examined the effect of melatonin endoplasmic reticulum (ER) stress and apoptosis of colorectal cancer cells.

\section{Materials and Methods}

Preparation of oxaliplatin. Oxaliplatin was purchased from Sigma (St. Louis, MO, USA). It was dissolved in dimethyl sulfoxide (DMSO), and aliquots were stored at $4^{\circ} \mathrm{C}$ until use.

Cell culture. The human colon cancer cell line (SNU-C5) and oxaliplatin-resistant cell line (SNU-C5/Oxal-R) were obtained from the Chosun University Research Center for Resistant Cells (Gwangju, Korea). SNU-C5 were cultured in RPMI 1640 (Thermo Fisher Scientific, Waltham, MA, USA) full medium, supplemented with $10 \%$ foetal bovine serum (FBS; Thermo Fisher Scientific), Lglutamine (Sigma), and antibiotics (Biological Industries, Beit Haemek, Israel). SUN-C5/Oxal-R were cultured in RPMI 1640 full medium without oxaliplatin $(14.7 \mu \mathrm{M})$ for 3 days, then cultured in fresh RPMI 1640 full medium with oxaliplatin $(14.7 \mu \mathrm{M})$ for 2 days. Both cell lines were incubated at $37^{\circ} \mathrm{C}$ with $5 \% \mathrm{CO}_{2}$ in a humidified condition.

Treatment of SNU-C5/Oxal-R cells. The SNU-C5/Oxal-R were washed twice with phosphate-buffered saline (PBS), and fresh RPMI-1640 medium containing 1\% FBS was added. Measurement of superoxide anions, antioxidant enzyme activity, and signalling pathway were investigated after pre-treating the cells with oxaliplatin $(1 \mu \mathrm{M})$.

Western blot analysis. Total cellular protein was extracted using RIPA lysis buffer (Thermo Fisher Scientific). Cell lysates were separated by sodium dodecyl sulfate-polyacrylamide gel electrophoresis, and the proteins were transferred to polyvinylidene fluoride membranes (Sigma). The membranes were blocked in 5\% skim milk and incubated with primary antibodies against $\mathrm{PrPC}$, phospho-protein kinase RNA-like endoplasmic reticulum kinase (p-PERK), PERK, phospho-inositol-requiring enzyme 1 (p-IRE1 $\alpha$ ), total IRE1 $\alpha$, activating transcription factor 4 (ATF4), CCAAT/enhancer-binding protein (CHOP), B-cell lymphoma 2 (BCL2), BCL2-associated X protein (BAX), cleaved caspase 3 (c-caspase 3), and $\beta$-actin (Santa Cruz Biotechnology, Santa Cruz, CA, USA). After incubation of the membranes with peroxidase-conjugated goat anti-mouse or anti-rabbit IgG secondary antibodies (Santa Cruz Biotechnology), the bands were visualized using enhanced chemiluminescence reagents (GE Healthcare, Chicago, IL, USA).

Dihydroethidium (DHE) staining. DHE (Sigma) was used to measure superoxide anion levels in cultured SNU-C5/Oxal-R cells. The cells were incubated with DHE $(10 \mu \mathrm{M})$ for $30 \mathrm{~min}$ at $37^{\circ} \mathrm{C}$. After washing with phosphate-buffered saline twice, the samples were analysed by Cyflow Cube 8 (Partec, Munster, Germany). Data analysis was performed using standard FSC Express (De Novo Software, Los Angeles, CA, USA).

Superoxide dismutase (SOD) activity. SNU-C5/Oxal-R cells were harvested from the culture dish by scraping with a rubber policeman on ice. The cells were extracted using extraction buffer. Cell lysates $(40 \mu \mathrm{g})$ generated superoxide by treatment with water-soluble tetrazolium salt (WST-1). The SOD activity was measured using an ELISA reader (BMG Labtech, Ortenberg, Germany) at an optical density of $450 \mathrm{~nm}$ for $10 \mathrm{~min}$.

Catalase activity. SNU-C5/Oxal-R cell lysate protein extracts $(30 \mu \mathrm{g})$ were incubated with $20 \mathrm{mM} \mathrm{H}_{2} \mathrm{O}_{2}$ (Sigma) in $0.1 \mathrm{M}$ Tris-HCl (Sigma) for $30 \mathrm{~min}$. Then, $50 \mathrm{mM}$ Amplex Red reagent (Thermo Fisher Scientific) and $0.2 \mathrm{U} / \mathrm{ml}$ horseradish peroxidase (Sigma) were added, and the samples were incubated for $15 \mathrm{~min}$ at $37^{\circ} \mathrm{C}$. Changes in the absorbance values associated with $\mathrm{H}_{2} \mathrm{O}_{2}$ degradation were measured using an ELISA reader (BMG Labtech) at $563 \mathrm{~nm}$.

Propidium iodide (PI)/annexin V flow cytometric analysis. SNU$\mathrm{C} 5 / \mathrm{Oxal}-\mathrm{R}$ cells were incubated in $60 \mathrm{~mm}$ cell culture plates and treated with oxaliplatin $(1 \mu \mathrm{M})$ or a combination of oxaliplatin (1 $\mu \mathrm{M})$ and melatonin $(500 \mu \mathrm{M})$ for $24 \mathrm{~h}$. The apoptosis of SNUC5/Oxal-R cells was assessed by Cyflow Cube 8 (Partec) after staining the cells with annexin V-fluorescein isothiocyanate and prodium iodide (PI) (Sigma). Data analysis was performed using standard FSC Express (De Novo Software).

Statistical analyses. Quantitative results are expressed as the mean \pm SEM. All experimental results were analysed by ANOVA or Student's $t$-test for paired comparisons. Differences were considered to be statistically significant when $p<0.05$.

\section{Results}

$\operatorname{PrP}^{C}$ may regulate intracellular superoxide anion generation in SNU-C5/Oxal-R cells. To investigate the expression of $\mathrm{PrP}^{\mathrm{C}}$ in wild-type and drug-resistant colorectal cancer cells, the level of $\mathrm{PrP}^{\mathrm{C}}$ in SNU-C5 and SNU-C5/Oxal-R cells was assessed by western blotting (Figure 1A). The expression of $\mathrm{PrP}^{\mathrm{C}}$ in SNUC5/Oxal-R cells was significantly increased compared with that in SNU-C5 cells (Figure 1B). To further determine whether $\mathrm{PrP}^{\mathrm{C}}$ regulates intracellular superoxide anion generation in SNU-C5 and SNU-C5/Oxal-R cells, superoxide anion generation was analysed by DHE staining (Figure 1C). The generation of superoxide anions in SNU-C5/Oxal-R cells was 
A

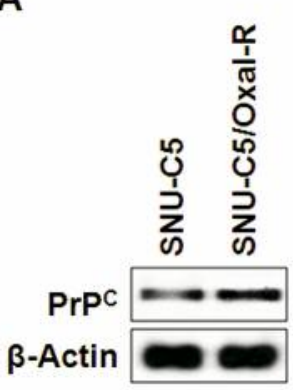

B

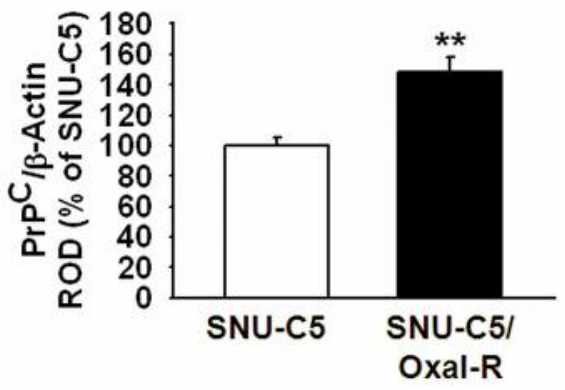

C

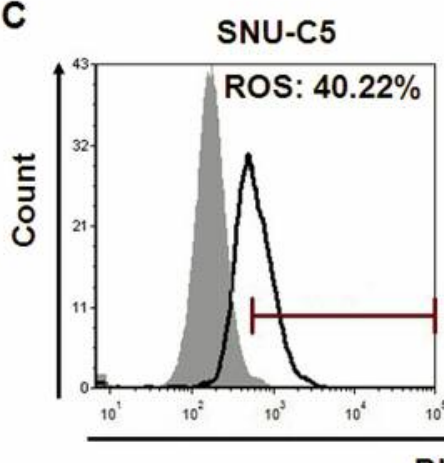

DHE

E

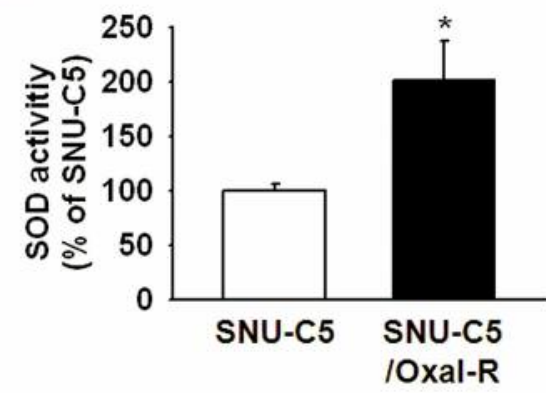

SNU-C5/Oxal-R

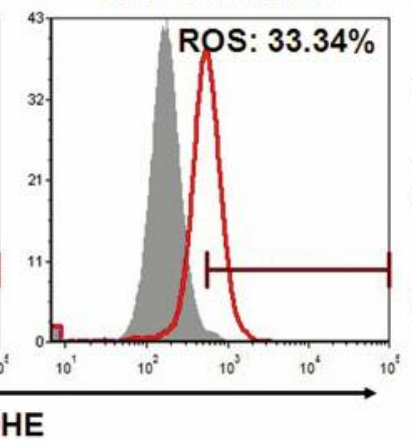

D

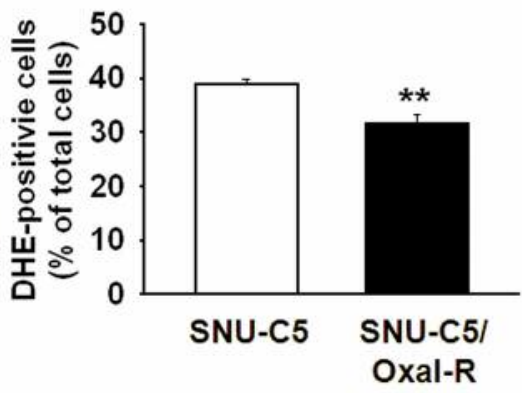

$\mathbf{F}$

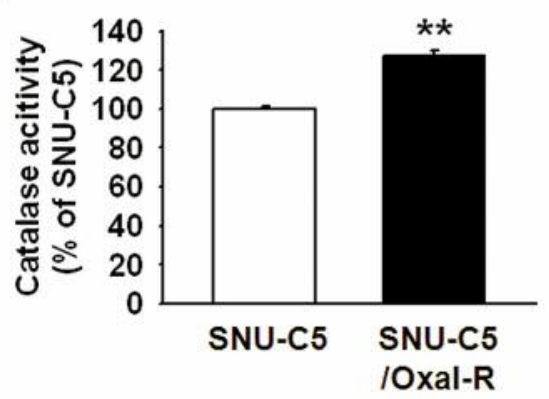

Figure 1. Anticancer drug-resistant SNU-C5/Oxal-R cells have lower generation of intracellular superoxide anion. A: Expression of cellular prion protein ( $\left.P r P^{C}\right)$ in SNU-C5 and SNU-C5/Oxal-R cells. B: The level of $\operatorname{Pr}^{C}$ was determined by densitometry relative to $\beta$-actin expression ( $\left.n=3\right)$. $C$ : Superoxide anion generation was measured by flow cytometric analysis of dihydroethidium (DHE) staining. D: Quantitative analysis of the percentage of DHE-positive cells by flow cytometric analysis (n=3). E: SOD activity of SNU-C5 and SNU-C5/Oxal-R cells $(n=3)$. F: Catalase activity of SNU-C5 and SNU-C5/Oxal-R cells ( $n=3)$. Data are expressed as the mean $\pm S E M$. Significantly different at $* p<0.05$ and $* * p<0.01 v s$. SNU-C5 cells.

significantly lower compared with that in SNU-C5 cells (Figure 1D). In addition, the activity of SOD and catalase was significantly increased in SNU-C5/Oxal-R cells. These results indicate that $\mathrm{PrP}^{\mathrm{C}}$ may be involved in regulation of the generation of superoxide anions in SNU-C5/Oxal-R cells through augmentation of SOD and catalase activities.

Melatonin may be involved in control of superoxide anions in SNU-C5/Oxal-R cells through regulation of $\mathrm{PrP}^{C}$ expression. To understand the effect of melatonin on $\operatorname{PrP}^{C}$ expression in drug-resistant colorectal cancer cells, the expression of $\mathrm{PrP}^{\mathrm{C}}$ in SNU-C5/Oxal-R cells treated with/without oxaliplatin with/without melatonin was analysed by western blotting (Figure 2A). Treatment with oxaliplatin did not significantly alter $\mathrm{PrP}^{\mathrm{C}}$ expression, whereas co-treatment with oxaliplatin and melatonin led to a significant decrease in $\operatorname{PrP}^{\mathrm{C}}$ expression (Figure 2B). In addition, treatment with oxaliplatin significantly increased the activity of SOD and catalase; however, these effects were blocked by treatment with melatonin (Figure 2C and D). Furthermore, superoxide anion 
A

B

SNU-C5/Oxal-R
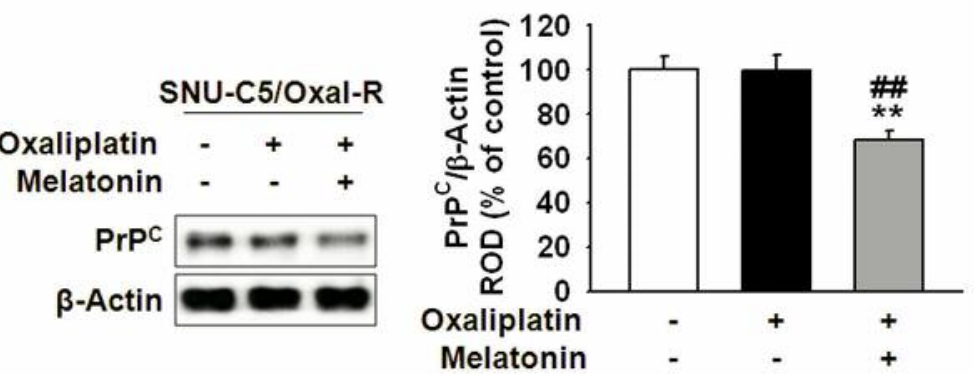

C

D
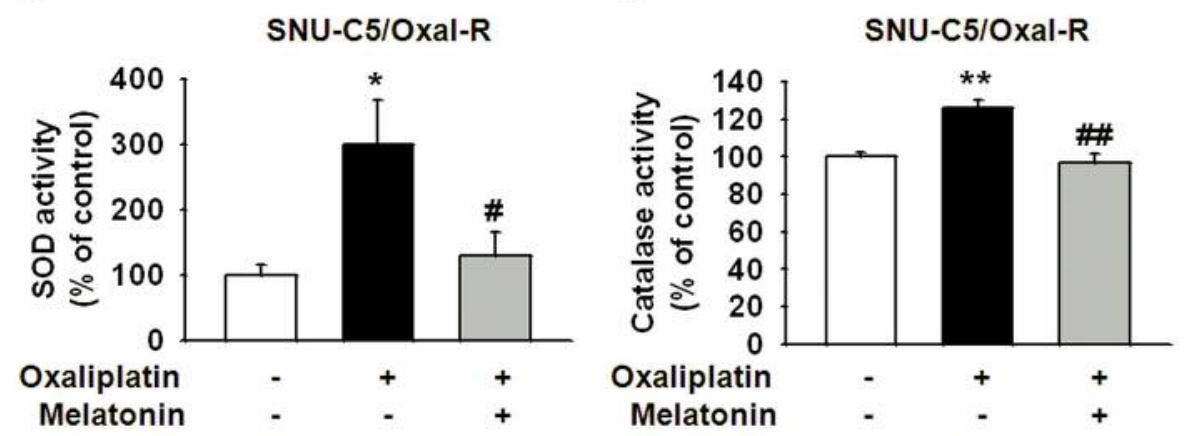

$\mathbf{E}$

SNU-C5/Oxal-R
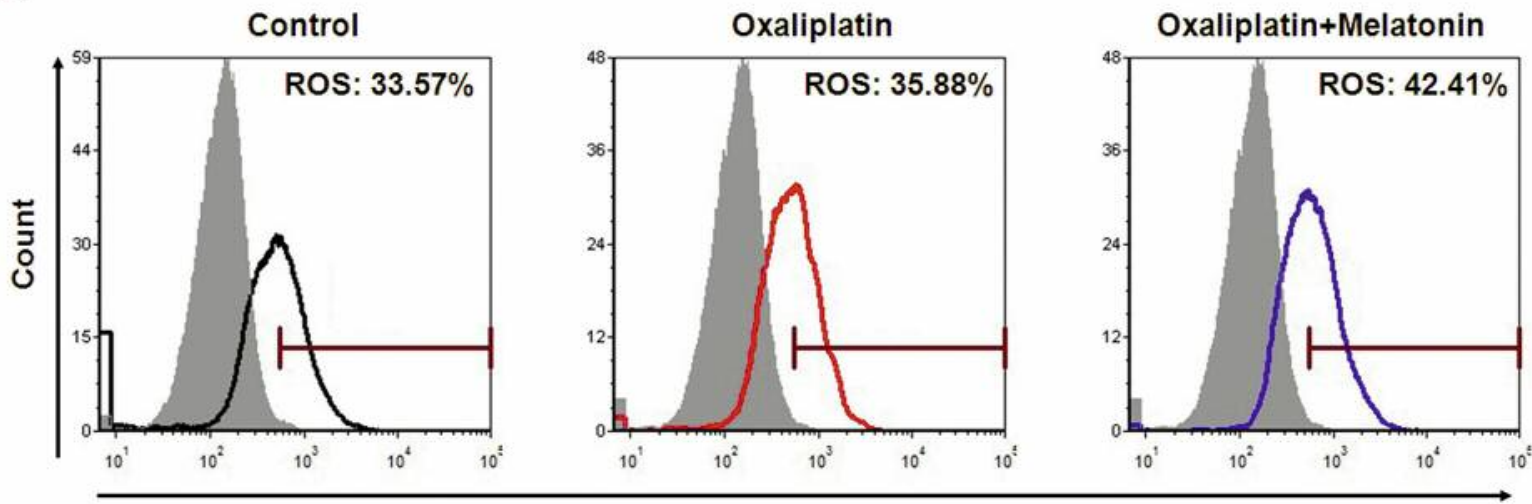

DHE

$\mathbf{F}$

\section{SNU-C5/Oxal-R}

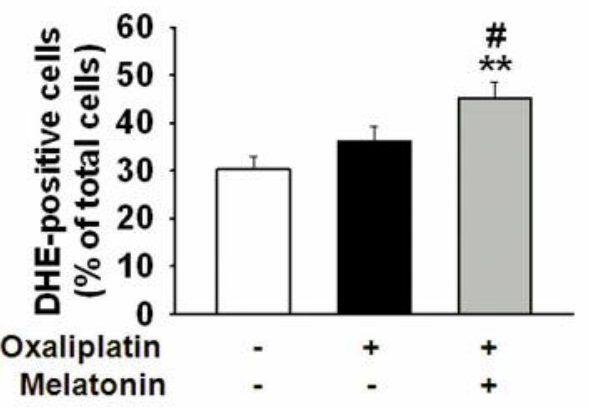

Figure 2. Effect of melatonin on cellular prion protein $(\operatorname{Pr} P C)$ expression and superoxide anion generation in SUN-C5/Oxal-R cells. A: Expression of $\mathrm{PrPC}^{\mathrm{C}}$ in SNU-C5/Oxal-R cells after treatment with/without oxaliplatin with/without melatonin. B: The level of $\operatorname{PrPC}^{C}$ was determined by densitometry relative to $\beta$-actin expression $(n=3) . C$ : Superoxide dismutase (SOD) activity of SNU-C5/Oxal-R cells treated with/without oxaliplatin with/without melatonin $(n=3)$. D: Catalase activity of SNU-C5/Oxal-R cells treated with/without oxaliplatin and/or melatonin $(n=3)$. E: Superoxide anion generation was measured by flow cytometric analysis of dihydroethidium (DHE) staining in SNU-C5/Oxal-R cells treated with/without oxaliplatin with/without melatonin. F: Quantitative analysis of the percentage of DHE-positive cells by flow cytometric analysis $(n=3)$. Data are expressed as the mean \pm SEM. Significantly different at: $* p<0.05$ and ${ }^{* *} p<0.01$ vs. non-treated cells; ${ }^{*} p<0.05$ and ${ }^{\# \#} p<0.01$ vs. oxaliplatin-treated cells. 
A
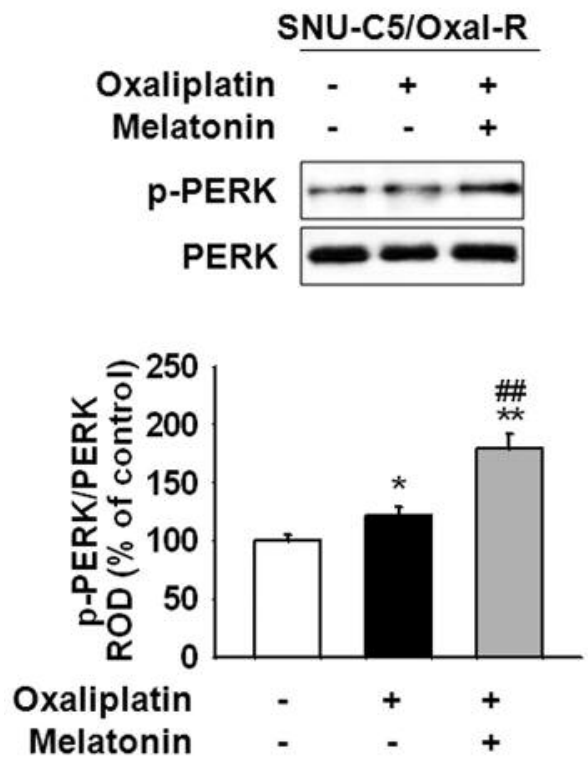

C

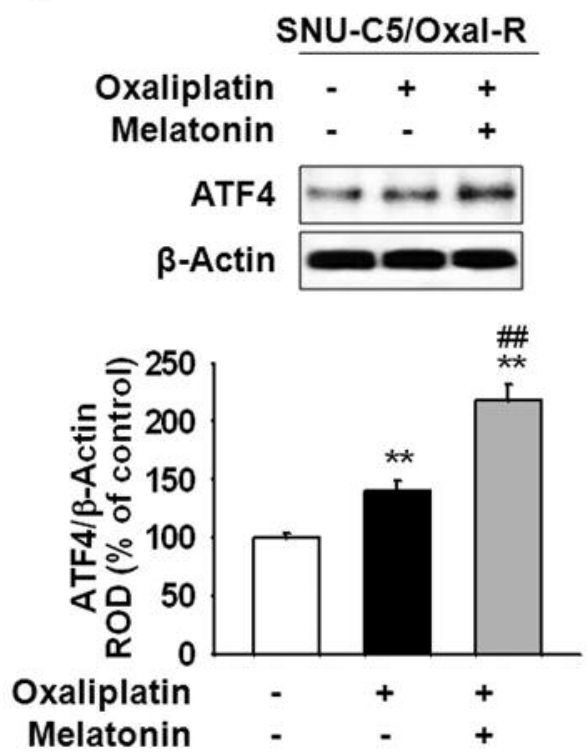

B

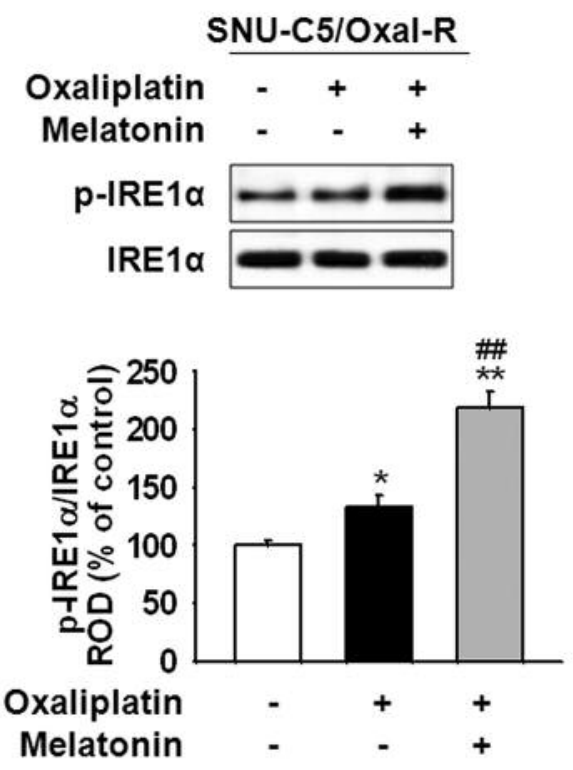

D

\section{SNU-C5/Oxal-R}
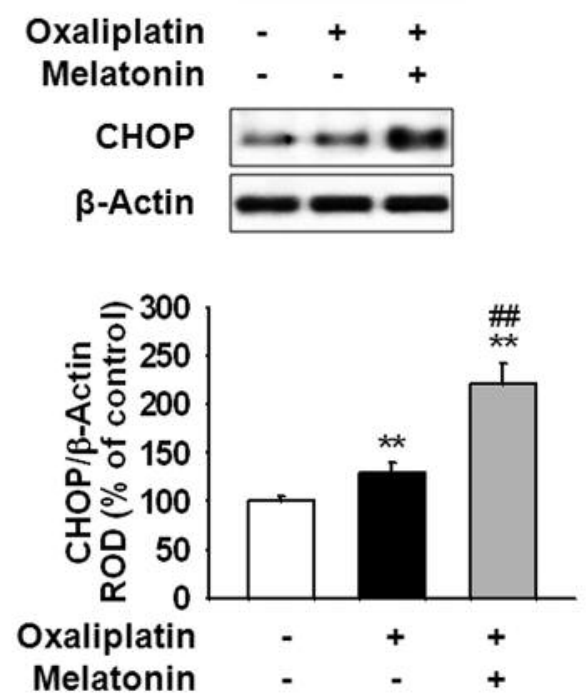

Figure 3. Effect of melatonin on oxaliplatin-induced endoplasmic reticulum (ER) stress in SNU-C5/Oxal-R cells. The level of phospho-protein kinase $R$-like endoplasmic reticulum kinase ( $p$-PERK) (A), phospho-inositol-requiring protein 1 alpha ( $p$-IRE1 $\alpha)(B)$, activating transcription factor 4 (ATF4) (C), and CCAAT-enhancer-binding protein homologous protein (CHOP) (D) was measured in SNU-C5/Oxal-R cells treated with/without oxaliplatin with/without melatonin by densitometry relative to total PERK, - -IRE1 $\alpha$, and $\beta$-actin expression, respectively $(n=3)$. Data are expressed as the mean \pm SEM. Significantly different at: ${ }^{*} p<0.05$ and ${ }^{*} p<0.01$ vs. non-treated cells; ${ }^{\# \#} p<0.01$ vs. oxaliplatin-treated cells.

generation in cells co-treated with oxaliplatin and melatonin was significantly increased compared with that in non-treated and oxaliplatin-treated cells (Figure 2E and F). These results demonstrated that melatonin appears to inhibit the activity of antioxidant enzymes in SNU-C5/Oxal-R cells through downregulation of $\mathrm{PrP}^{\mathrm{C}}$ expression.
Melatonin triggers endoplasmic reticulum (ER) stress in $S N U-C 5 / O x a l-R$ cells. To investigate the effect of oxaliplatin on ER stress in drug-resistant colorectal cancer cells, the activation of ER stress-associated proteins PERK, IRE1 $\alpha$, ATF4, and CHOP was assessed by western blotting after treating SNU-C5/Oxal-R cells with oxaliplatin. PERK, 
A
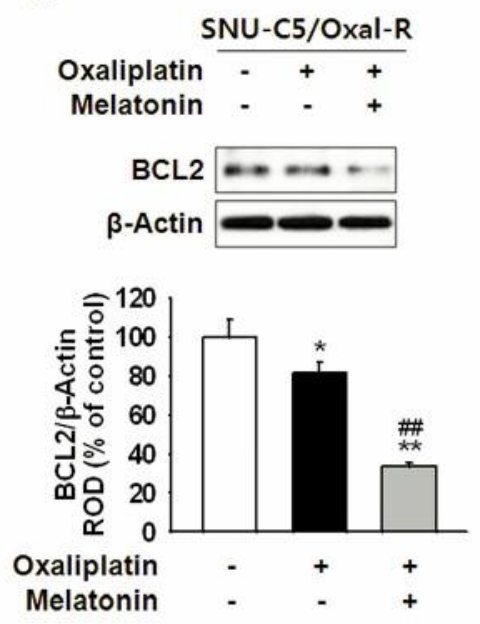

B
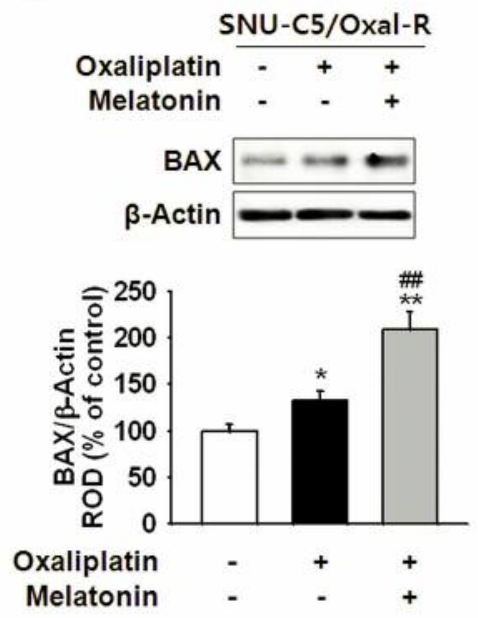

C
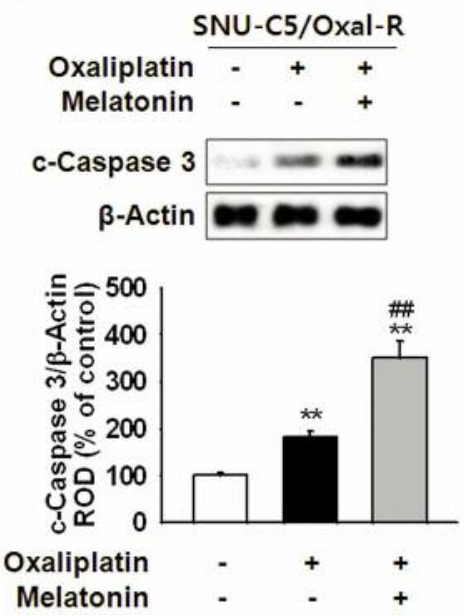

D

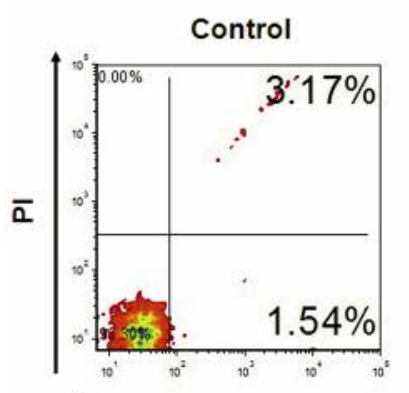

SNU-C5/Oxal-R

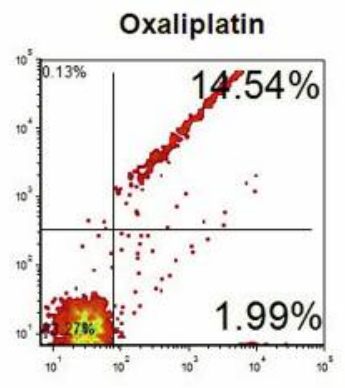

Oxaliplatin+Melatonin

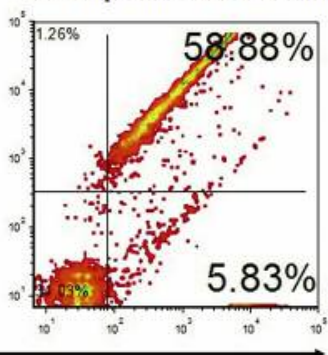

Annexin V

E

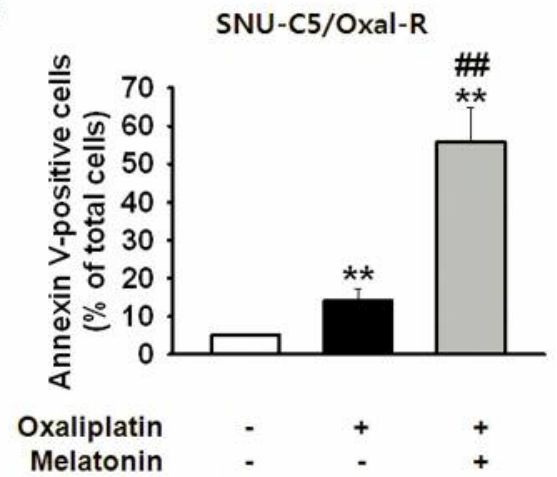

Figure 4. Effect of melatonin on oxaliplatin-induced apoptosis of SNU-C5/Oxal-R cells. The expression of B-cell lymphoma 2 (BCL2) (A), BCL2associated X protein $(B A X)(B)$, and cleaved caspase 3 (c-caspase 3) (C) in SNU-C5/Oxal-R cells treated with/without oxaliplatin with/without melatonin was determined by densitometry relative to $\beta$-actin expression $(n=3)$. D: Apoptosis was measured by flow cytometric analysis of PI/Annexin V staining in SNU-C5/Oxal-R cells treated with/without oxaliplatin and/or melatonin. E: Quantitative analysis of the percentage of apoptotic cells by flow cytometric analysis $(n=3)$. Data are expressed as the mean \pm SEM. Significantly different at: ${ }^{*} p<0.05$ and $* * p<0.01$ vs. nontreated cells; ${ }^{\# \#} p<0.01$ vs. oxaliplatin-treated cells.

IRE1 $\alpha$, ATF4, and CHOP levels in oxaliplatin-treated cells were significantly increased compared with those in nontreated cells (Figure 3). Interestingly, the levels of these proteins in cells co-treated with melatonin and oxaliplatin were further increased compared with those in cells treated only with oxaliplatin (Figure 3 ). These findings indicate that melatonin appears to trigger ER stress in SNU-C5/Oxal-R cells following drug treatment.

Melatonin induces oxaliplatin-mediated apoptosis of SNU-C5/Oxal-R cells. To examine whether melatonin is involved in drug-mediated apoptosis of drug-resistant colorectal cancer cells, the expression levels of apoptosis-mediated proteins BCL2, BAX, and caspase-3 in SNU-C5/Oxal-R cells were analysed by western blotting after treatment with oxaliplatin. The expression of the anti-apoptotic protein BCL2 in oxaliplatintreated cells was significantly reduced compared with that in non-treated cells (Figure 4A). The levels of pro-apoptotic proteins (BAX and c-caspase 3) in oxaliplatin-treated cells were significantly increased compared with those in nontreated cells (Figure 4B and C). In comparison with the expression of BCL2, BAX, and c-caspase 3 in cells treated only with oxaliplatin, the expression of BCL2 was reduced and that of BAX and c-caspase 3 was increased in cells cotreated with melatoninin combination with oxaliplatin (Figure 4A-C). In addition, PI/annexin V staining showed that the apoptosis of co-treated cells was enhanced compared 
with that of non-treated cells and cells treated with oxaliplatin alone (Figure 4D and E). These results demonstrate that melatonin appears to induce oxaliplatinmediated apoptosis of SNU-C5/Oxal-R cells.

\section{Discussion}

This study demonstrated that the expression of $\mathrm{PrP}^{\mathrm{C}}$ was increased in SNU-C5/Oxal-R cells, and SNU-C5 cells increased the activity of antioxidant enzymes (SOD and catalase) and reduced generation of superoxide anions compared with SNU-C5 cells. In the presence of oxaliplatin, the level of $\mathrm{PrP}^{\mathrm{C}}$ in SNU-C5/Oxal-R cells was not significantly different, although the levels of antioxidants were significantly increased. However, treatment with melatonin reduced the level of $\operatorname{PrP}^{\mathrm{C}}$, and resulted in the inhibition of the activity of antioxidant enzymes in SNUC5/Oxal-R cells. In addition, treatment of SNU-C5/Oxal-R cells with melatonin induced ER stress and apoptosis.

Normal $\mathrm{PrP}^{\mathrm{C}}$ is a ubiquitous glycoprotein that is involved in various physiological cellular processes, including proliferation, differentiation, stress protection, and signal transduction regulation (22). $\operatorname{PrP}^{\mathrm{C}}$ silencing can also increase sensitivity to oxidative stress by reducing SOD activity (23). In addition, the activity of antioxidant enzymes, including catalase and glutathione reductase, has been reported to be reduced in Prnp knockout mice $(24,25)$. This study revealed that the level of $\mathrm{PrP}^{\mathrm{C}}$ was increased in SNU-C5/Oxal-R cells. Furthermore, SNU-C5/Oxal-R cells exhibited a higher antioxidant effect through the enhancement of SOD and catalase activities. A recent study has reported that $\operatorname{PrP}^{\mathrm{C}}$ participates in the multidrug resistance of gastric cancer (26). Overexpression of $\operatorname{PrP}^{\mathrm{C}}$ has been found to increase resistance to doxorubicin in colorectal cancer cells (27). Moreover, our previous study revealed that $\mathrm{PrP}^{\mathrm{C}}$ promoted 5-fluorouracil resistance of colorectal cancer cells (12). These findings suggest that $\mathrm{PrP}^{\mathrm{C}}$ may be an important molecule in anticancer drug resistance.

In this study, we revealed that co-treatment with oxaliplatin and melatonin increased ER stress in and apoptosis of SNUC5/Oxal-R cells. Several studies have demonstrated the anticancer effect of melatonin on various cancer types, such as breast, prostatic, ovarian, cervical, endometrial, renal, lung, gastric, pancreatic, and colorectal cancer (18). In colorectal cancer cells, melatonin was found to increase the cellular ROS level, leading to cell death (19). Treatment with melatonin induced apoptosis, autophagy, and senescence in colorectal cancer cells through the induction of $G_{1}$ phase arrest (21). Melatonin can also induce mitochondria-mediated apoptosis (28). Furthermore, co-treatment of the colorectal cancer cell lines SW480 and LoVo with anticancer agents, ursolic acid, and melatonin has been reported to facilitate apoptosis via the cytochrome c/caspase signalling pathway (29). Our findings show that co-treatment with oxaliplatin and melatonin reduced the level of $\mathrm{PrP}^{\mathrm{C}}$ and led to the suppression of antioxidant enzyme activity and the augmentation of superoxide anion generation. The increase in superoxide anion generation appears to have led to the activation of the ER stress-mediated signalling pathway and induced apoptosis via the regulation of apoptosis-associated proteins. These results indicate that melatonin increased ER stress and apoptosis of SNU-C5/Oxal-R cells possibly through inhibition of $\operatorname{PrP}^{\mathrm{C}}$ expression.

Overall, this study showed that $\operatorname{PrP}^{\mathrm{C}}$ expression is increased in SNU-C5/Oxal-R cells compared to their parental cells. In addition, $\mathrm{PrP}^{\mathrm{C}}$ may play a key role in the activity of antioxidant enzymes in SNU-C5/Oxal-R cells. We revealed that co-treatment with oxaliplatin and melatonin increased apoptosis of SNU-C5/Oxal-R cells, thus overcoming the oxaliplatin resistance of colorectal cancer cells.

In addition, we suggested from previous results that $\operatorname{PrPC}^{\mathrm{C}}$ plays an important role in anticancer-resistant cells $(12,30)$ and is related to an antioxidant function $(31,32)$. In conclusion, this study showed that SNU-C5/Oxal-R cells had increased expression of $\mathrm{PrP}^{\mathrm{C}}$ compared to SNU-C5 cells. Moreover, co-treatment with anticancer drugs and melatonin may be an effective strategy for patients with drug-resistant colorectal cancer.

\section{Conflicts of Interest}

The Authors have no conflicts of interest to declare with regards to this study.

\section{Acknowledgements}

This work was supported by the National Research Foundation grant funded by the Korean government (grant number NRF2016R1D1A3B01007727, NRF-2017M3A9B4032528). The funding agencies had no role in formulating the study design, data collection or analysis, the decision to publish, or preparation of the manuscript.

\section{References}

1 Siegel RL, Miller KD and Jemal A: Cancer Statistics, 2017. CA Cancer J Clin 67: 7-30, 2017.

2 Kelland L: The resurgence of platinum-based cancer chemotherapy. Nat Rev Cancer 7: 573-584, 2007.

3 Holohan C, Van Schaeybroeck S, Longley DB and Johnston PG: Cancer drug resistance: an evolving paradigm. Nat Rev Cancer 13: 714-726, 2013.

4 Liang J and Kong Q: Alpha-cleavage of cellular prion protein. Prion 6: 453-460, 2012.

5 Lee JH, Han YS and Lee SH: Potentiation of biological effects of mesenchymal stem cells in ischemic conditions by melatonin via up-regulation of cellular prion protein expression. J Pineal research 62, 2017. doi: 10.1111/jpi.12385

6 Shi F, Yang Y, Wang T, Kouadir M, Zhao D and Hu S: Cellular prion protein promotes neuronal differentiation of adiposederived stem cells by up-regulating miRNA-124. J Mol Neurosci 
59: 48-55, 2016.

7 Doeppner TR, Kaltwasser B, Schlechter J, Jaschke J, Kilic E, Bahr M, Hermann DM and Weise J: Cellular prion protein promotes post-ischemic neuronal survival, angioneurogenesis and enhances neural progenitor cell homing via proteasome inhibition. Cell Death Dis 6: e2024, 2015.

8 Martin-Lanneree S, Hirsch TZ, Hernandez-Rapp J, Halliez S, Vilotte JL, Launay JM and Mouillet-Richard S: $\operatorname{PrP}(\mathrm{C})$ from stem cells to cancer. Front Cell Dev Biol 2: 55, 2014.

9 Li QQ, Sun YP, Ruan CP, Xu XY, Ge JH, He J, Xu ZD, Wang $\mathrm{Q}$ and Gao WC: Cellular prion protein promotes glucose uptake through the Fyn-HIF-2alpha-Glut1 pathway to support colorectal cancer cell survival. Cancer Sci 102: 400-406, 2011.

10 Wang Q, Qian J, Wang F and Ma Z: Cellular prion protein accelerates colorectal cancer metastasis via the FYN-SP1SATB1 axis. Oncology Rep 28: 2029-2034, 2012.

11 Hinton C, Antony H, Hashimi SM, Munn A and Wei MQ: Significance of prion and prion-like proteins in cancer development, progression and multi-drug resistance. Current Cancer Drug Targ 13: 895-904, 2013.

12 Lee JH, Yun CW and Lee SH: Cellular prion protein enhances drug resistance of colorectal cancer cells via regulation of a survival signal pathway. Biomol Ther 26: 1-9, 2018.

13 Garcia JJ, Lopez-Pingarron L, Almeida-Souza P, Tres A, Escudero P, Garcia-Gil FA, Tan DX, Reiter RJ, Ramirez JM and Bernal-Perez M: Protective effects of melatonin in reducing oxidative stress and in preserving the fluidity of biological membranes: a review. J Pineal Res 56: 225-237, 2014.

14 Jan JE, Reiter RJ, Wasdell MB and Bax M: The role of the thalamus in sleep, pineal melatonin production, and circadian rhythm sleep disorders. J Pineal Res 46: 1-7, 2009.

15 Reiter RJ, Tan DX, Manchester LC, Paredes SD, Mayo JC and Sainz RM: Melatonin and reproduction revisited. Biol Reprod 81: 445-456, 2009.

16 Fernandez A, Ordonez R, Reiter RJ, Gonzalez-Gallego J and Mauriz JL: Melatonin and endoplasmic reticulum stress: relation to autophagy and apoptosis. J Pineal Res 59: 292-307, 2015.

17 Reiter RJ, Mayo JC, Tan DX, Sainz RM, Alatorre-Jimenez M and Qin L: Melatonin as an antioxidant: under promises but over delivers. J Pineal Res 61: 253-278, 2016.

18 Li Y, Li S, Zhou Y, Meng X, Zhang JJ, Xu DP and Li HB: Melatonin for the prevention and treatment of cancer. Oncotarget 8: 39896-39921, 2017.

19 Buldak RJ, Pilc-Gumula K, Buldak L, Witkowska D, Kukla M, Polaniak R and Zwirska-Korczala K: Effects of ghrelin, leptin and melatonin on the levels of reactive oxygen species, antioxidant enzyme activity and viability of the HCT 116 human colorectal carcinoma cell line. Mol Med Rep 12: 2275-2282, 2015.

20 Wei JY, Li WM, Zhou LL, Lu QN and He W: Melatonin induces apoptosis of colorectal cancer cells through HDAC4 nuclear import mediated by CaMKII inactivation. J Pineal Res 58: 429438, 2015.

21 Hong Y, Won J, Lee Y, Lee S, Park K, Chang KT and Hong Y: Melatonin treatment induces interplay of apoptosis, autophagy, and senescence in human colorectal cancer cells. J Pineal Res 56: 264-274, 2014.
22 Castle AR and Gill AC: Physiological functions of the cellular prion protein. Front Mol Biosci 4: 19, 2017.

23 Brown DR, Schulz-Schaeffer WJ, Schmidt B and Kretzschmar HA: Prion protein-deficient cells show altered response to oxidative stress due to decreased SOD-1 activity. Exp Neurol 146: 104-112, 1997.

24 Brown DR, Nicholas RS and Canevari L: Lack of prion protein expression results in a neuronal phenotype sensitive to stress. J Neurosci Res 67: 211-224, 2002.

25 Klamt F, Dal-Pizzol F, Conte da Frota ML Jr., Walz R, Andrades ME, da Silva EG, Brentani RR, Izquierdo I and Fonseca Moreira JC: Imbalance of antioxidant defense in mice lacking cellular prion protein. Free Rad Biol Med 30: 1137-1144, 2001.

26 Luo G, Wang W, Wu Q, Lu Y, Su T, Gu N, Li K, Wang J, Du R, Zhao X, Li X, Fan R, Zhang H, Nie Y, Zhou X, Shi Y, Liang J, Wang $X$ and Fan D: MGr1-Antigen/37 kDa laminin receptor precursor promotes cellular prion protein induced multi-drugresistance of gastric cancer. Oncotarget 8: 71630-71641, 2017.

27 Chieng CK and Say YH: Cellular prion protein contributes to LS $174 \mathrm{~T}$ colon cancer cell carcinogenesis by increasing invasiveness and resistance against doxorubicin-induced apoptosis. Tumour Biol 36: 8107-8120, 2015.

28 Bejarano I, Redondo PC, Espino J, Rosado JA, Paredes SD, Barriga C, Reiter RJ, Pariente JA and Rodriguez AB: Melatonin induces mitochondrial-mediated apoptosis in human myeloid HL-60 cells. J Pineal Res 46: 392-400, 2009.

29 Wang J, Guo W, Chen W, Yu W, Tian Y, Fu L, Shi D, Tong B, Xiao X, Huang W and Deng W: Melatonin potentiates the antiproliferative and pro-apoptotic effects of ursolic acid in colon cancer cells by modulating multiple signaling pathways. $\mathrm{J}$ Pineal Res 54: 406-416, 2013.

30 Lee JH, Han YS, Yoon YM, Yun CW, Yun SP, Kim SM, Kwon HY, Jeong D, Baek MJ, Lee HJ, Lee SJ, Han HJ and Lee SH: Role of HSPA1L as a cellular prion protein stabilizer in tumor progression via HIF-1alpha/GP78 axis. Oncogene 36: 6555$6567,2017$.

31 Han YS, Lee JH, Yoon YM, Yun CW, Noh H and Lee SH: Hypoxia-induced expression of cellular prion protein improves the therapeutic potential of mesenchymal stem cells. Cell Death Dis 7: e2395, 2016.

32 Yoon YM, Lee JH, Yun SP, Han YS, Yun CW, Lee HJ, Noh H, Lee SJ, Han HJ and Lee SH: Tauro-ursodeoxycholic acid reduces ER stress by regulating of Akt-dependent cellular prion protein. Scientific Rep 6: 39838, 2016.
Received February 5, 2018

Revised February 27, 2018

Accepted March 6, 2018 